\title{
Microchannel Electrode Stimulation of Deep Peroneal Nerve Fascicles Induced Mean Arterial Depressor Response in Hypertensive Rats
}

\author{
Young-tae Kim, ${ }^{1,2^{*}}$ Aswini Kanneganti, ${ }^{3^{*}}$ Caleb Nothnagle, ${ }^{4}$ Ryan Landrith, ${ }^{1,4}$ Masaki Mizuno, \\ Muthu B J Wijesundara, ${ }^{4}$ Scott Smith, ${ }^{5}$ and Mario I Romero-Ortega,
}

${ }^{1}$ Department of Bioengineering, University of Texas at Arlington, Arlington, Texas, United States of America; ${ }^{2}$ Department of Urology, University of Texas Southwestern Medical Center, Dallas, Texas, United States of America; ${ }^{3}$ Department of Bioengineering, University of Texas at Dallas, Richardson, Texas, United States of America; ${ }^{4}$ University of Texas at Arlington Research Institute, University of Texas at Arlington, Fort Worth, Texas; ${ }^{5}$ Department of Health Care Sciences and Internal Medicine, University of Texas Southwestern Medical Center, Dallas, Texas, United States of America; and ${ }^{6}$ Department of Surgery, University of Texas Southwestern Medical Center, Dallas, Texas, United States of America

\begin{abstract}
Hypertension (HTN) affects over 1 billion people in the world, and while most are treated effectively with pharmacological regimens, $10-30 \%$ of them do not show a beneficial response. Electrical stimulation of the renal sympathetic, vagus and carotid sinus nerves has shown depressor effects and has been proposed as an alternative treatment for resistant hypertension (R-HTN). However, these nerves are heterogeneous in afferent/efferent composition, and their stimulation often results in unwanted side effects. We evaluated the possibility of eliciting a depressor response from stimulation of single fascicle of the somatic deep peroneal nerve (fDPN). A microchannel electrode array ( $\mu$ CEA) was used to stimulate the fDPN at low frequency, which induced a significant $(p \leq 0.03)$ transient reduction in mean arterial pressure (MAP) with no significant effects on heart rate. The depressor response was prolonged for several hours by extending the fDPN stimulation to $5 \mathrm{~min}$, which induced significant reduction (17-25\%) in MAP for up to $4 \mathrm{~h}$. Immunofluorescence evaluation of the axonal marker, myelin, and active macrophages in the fDPN revealed no indication of nerve damage or overt inflammation in response to the procedure. This study provides evidence supporting the use of $\mu$ CEA interfacing of small somatic nerve fascicles associated with cardiovascular relevant acupoints to induce significant reductions in MAP and opens the possibility of neuromodulation of small fascicles as an alternative strategy to treat R-HTN with minimal side effects. Further, the $\mu$ CEA multielectrode array offers an effective tool for neuromodulation of small nerve fascicles, enabling a number of possible future medical bioelectronic applications.
\end{abstract}

Online address: www.bioelecmed.org

doi: 10.15424/bioelectronmed.2015.00001

\section{INTRODUCTION}

Hypertension (HTN) affects over 1 billion people around the world (1). The current management of hypertension relies on thiazide-type diuretics, calcium

*YK and AK contributed equally to the study.

Address correspondence to Mario I Romero-Ortega, Department of Bioengineering, University of Texas at Dallas, Richardson, TX 75080. Phone: 972-883-6824; Fax: 972-883-2710; E-mail: mario.romero-ortega@utdallas.edu; or Young-tae Kim, Department of Bioengineering, University of Texas at Arlington, Arlington, TX 76010. Phone: 817-272-5023; Fax: 817-2722251; E-mail: ykim@uta.edu.

Submitted January 22, 2015; Accepted for publication August 26, 2015; Published Online (www.bioelecmed.org) August 28, 2015.

\section{The Feinstein Institute for Medical Research} Empowering Imagination. Pioneering Discovery. channel blockers, angiotensin-converting enzyme inhibitors and/or angiotensin receptor blockers to maintain normal blood pressure and prevent myocardial infarction, stroke, renal failure and death (2).
However, appropriate dosing is challenging because of the high variability in the patient response, narrow therapeutically acceptable range, adverse reactions and toxicity (3). Moreover, approximately $10-30 \%$ of the affected population is resistant to pharmacological treatments, despite the use of combinatorial drug treatments and maximum doses $(4,5)$. Individuals suffering from resistant hypertension (R-HTN) have high rates of cardiovascular complications and few treatment options. Unfortunately, some promising research candidates for the treatment of R-HTN such as renal artery denervation have failed to show efficacy $(6,7)$. Therefore, there is much need for developing alternative methods for $\mathrm{R}-\mathrm{HTN}$ regulation. 
Vagal nerve stimulation (VNS) was first proposed as a possible method for the treatment of HTN in the late 1990s, but reports of bradycardia and apnea side effects limited its application (8). Recent studies have shown that selective VNS using tripolar cuff electrode stimulation reduces blood pressure from 100 to $80 \mathrm{mmHg}$ in normal rats with minimal side effects (that is, $5-10 \%$ in apnea) using $40 \mathrm{~Hz}, 1 \mathrm{~mA}$ and 0.3-ms pulse stimulation for $4 \mathrm{~ms}$ (135 pulses) (9). However, apnea increased to $15 \%$ when stimulation was applied at $50 \mathrm{~Hz}$. Furthermore, whether this approach can achieve the same therapeutic effect in hypertensive animals was not investigated. In addition, vagal nerve is highly heterogeneous in axonal composition and side effects including cough, hoarseness and voice alteration, and paresthesias have been reported in humans $(9,10)$. Thus, alternative peripheral nerves that can achieve regulation of HTN with significantly less side effects might offer a more appealing treatment for R-HTN.

A recent randomized blind clinical trial demonstrated that active traditional Chinese acupuncture significantly lowers blood pressure in HTN patients compared with sham acupuncture (11), and some report the effect of acupuncture and pharmacological treatment (12). While the working mechanism of this therapeutic effect is unknown, increasing evidence suggests that the acupuncture therapy benefit is based on neuromodulation of the underlying nervous system. Indeed, depressor responses of $\sim 20 \mathrm{mmHg}$ have been reported in hypertensive rats after stimulation of the sciatic nerve (13). Recently, it was shown that electropuncture on a fascicle of the sciatic nerve (the common peroneal nerve, which overlays the acupoint Zuzanli [ST36] and Shangjuxu [ST37]) significantly reduces blood pressure $10-18 \mathrm{mmHg}$ without affecting heart rate (HR) (14).

We hypothesized that electrical stimulation of a fascicle of the somatic deep peroneal nerve (fDPN) provides an effective substrate to induce a significant depressor response in HTN with minimal

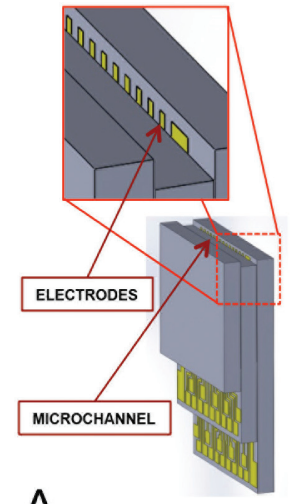

A



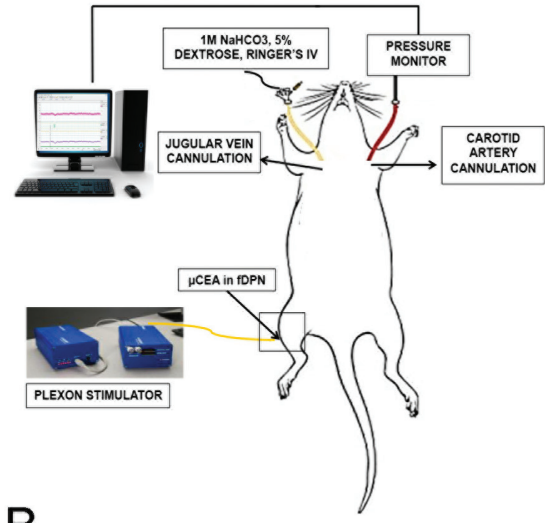

B

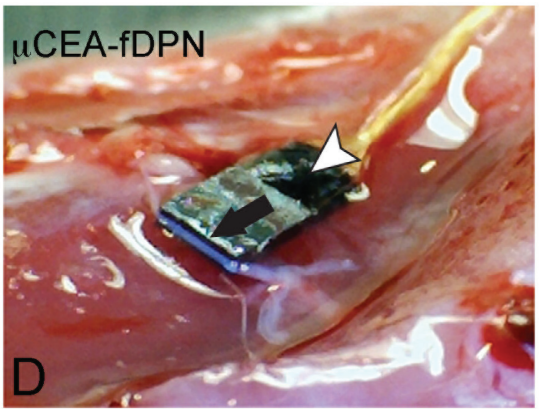

Figure 1. $\mu$ CEA interfacing of the fDPN. (A) Schematic of the $\mu$ CEA designed to interface small nerves (50-300 $\mu \mathrm{m}$ OD). (B) Illustration of the experimental animal setup. The fDPN was placed in the $\mu$ CEA electrode, and the wire connected to a stimulator box and a computer interface. A catheter in the jugular vein was used to replenish liquids, and cannulation of the carotid artery allowed recording of MAP during fascicular stimulation. (C) Photograph showing the isolation of a single fDPN fascicle (black arrow). (D) Placement of the fDPN (black arrow) in the groove of the $\mu$ CEA (arrowhead). Scale bar $=1 \mathrm{~mm}$.

side effects. A microchannel electrode array ( $\mu$ CEA) specifically designed and fabricated for interfacing with small nerves (that is, 50-300 $\mu \mathrm{m}$ in diameter; autonomic nerve or nerve fascicles) was used to stimulate the fDPN $(\sim 150 \mu \mathrm{m}$ in diameter) (15). We demonstrate that a significant reduction in mean arterial pressure (MAP) in hypertensive rats can be achieved through microelectrical stimulation of a somatic nerve fascicle for up to $4 \mathrm{~h}$ without significantly altering HR.

\section{MATERIALS AND METHODS}

\section{Microchannel Electrode Array ( $\mu$ CEA)}

Large diameter nerves such as the vagus (1-1.5 $\mathrm{mm}$ in a rat) are commonly interfaced with cuff electrodes. Because of their size and their tough epineurium, they withstand electrode implantation procedures that include pulling and stretching the nerve. Conversely, small diameter nerves or fascicles have a thinner epineurium or only perineurium and thus are more susceptible to injury during electrode implantation and chronic testing with current cuff designs. We have previously developed a $\mu$ CEA to facilitate the interface with small diameter nerves (50-300 $\mu \mathrm{m})$ with minimal impact on the nerve during implantation. The $\mu C E A$ electrode was fabricated and characterized as reported previously (15). Briefly, silicon dyes were stacked to form a 2-mm microchannel (that is, $200 \mu \mathrm{m}$ wide), where 15 gold square electrodes $\left(40 \times 50 \mu \mathrm{m}\right.$; approximately 2,000 $\left.\mathrm{m}^{2}\right)$ were placed bilaterally on the sidewalls (100 $\mu \mathrm{m}$ tall; Figure 1A). These dimen- 
sions were optimized for reading the neural output of a small diameter nerve in a rat. The $\mu$ CEA was coupled to an Omnetics connector by using sonically bonded Parylene-C insulated 25- $\mu$ m gold wires and further insulated with epoxy (15). Impedance spectroscopy showed values $<500 \mathrm{k} \Omega$ in at least $50 \%$ of the electrodes.

\section{Acute $\mu$ CEA Implantation onto the Ventral Fascicle of the DPN}

Six normotensive Wistar Kyoto (WKY, $\mathrm{n}=6$ ) rats and nine spontaneously hypertensive rats (SHR, Harlan, $n=9$ ) were used in this study. Because hypertensive SHR and normotensive WKY rats were both established from the same parental line, WKY rats were used as control rats in studies of SHR (16). The animals were anesthetized with inhaled isoflurane (1-3\% in $100 \%$ oxygen) from a vaporizer with a scavenger system. An appropriate level of anesthesia was confirmed by the lack of response to noxious stimuli. As previously described (17), intubation via tracheotomy and cannulation of the left carotid artery and right jugular vein was performed. The arterial cannula was interfaced with a flow pressure sensor (Model DTX plus-DT, NN12, Ohmeda) for continuous MAP and HR monitoring (Figure 1B). To stabilize fluid balance and maintain baseline MAP, a continuous infusion of physiological saline $(2 \mathrm{~mL}$, $1 \mathrm{~mol} / \mathrm{L} \mathrm{NaHCO}_{3} ; 10 \mathrm{~mL}$ \% dextrose) was administered into the jugular vein (3-5 $\left.\mathrm{mL} \cdot \mathrm{h}^{-1} \cdot \mathrm{kg}^{-1}\right)$.

The DPN was exposed in the right hind limb by lateral spreading of the gastrocnemius and peroneus muscles, and a 150-200 $\mu \mathrm{m}$ diameter ventral fascicle (fDPN) was gently separated with a glass rod (Figures 1C, D). The fascicle was then easily inserted into the groove of the $\mu \mathrm{CEA}$ and secured by using a medical grade silicone elastomer (Kwik-Sil, World Precision Instruments).

Animals were then transferred to urethane-based anesthesia and stabilized for $1 \mathrm{~h}$ before stimulation, and arterial blood gases and body temperature were maintained at normal values throughout the experiment. The UTSW Institutional Animal Care and Use Committee (IACUC) approved all procedures, and the studies were conducted in accordance with the Guide for the Care and Use of Laboratory Animals, used by the U.S. Department of Health and Human Services National Institutes of Health.

\section{Short- and Long-Duration fDPN Electrical Stimulation}

We first evaluated the response of the entire DPN to electrical stimulation for the MAP depressor response in WKY and SHR animals by using custom-made hooks with multistranded stainless steel wires (Bioflex wire AS633, Cooner Wire, $\mathrm{CA})$, to determine the optimal range of current intensities (0.1, 0.3 and $0.5 \mathrm{~mA})$, number of pulses ( 1 and 10 pulses) and stimulation frequency ( 1 and $100 \mathrm{~Hz}$ ).

Subsequently, a set of identified conditions $(0.1 \mathrm{~mA}, 10$ pulses, $1-2 \mathrm{~Hz})$ were used to stimulate the fDPN by using the $\mu C E A$ either for $10 \mathrm{~s}$ (short duration, $\mathrm{n}=$ 6 SHR and WKY) or 5 min (long duration, $\mathrm{n}=3 \mathrm{SHR})$. The biphasic cathodic leading current stimulation pulses were given by using a Plexon Stimulator module (Plexon), and the resultant effects were monitored continuously for either $40 \mathrm{~s}$ (short duration) or up to $4 \mathrm{~h}$ after stimulation. For consecutive trials, a minimum of 15-20 min of recovery time was maintained to (a) avoid crosscontamination between trials and (b) stabilize the MAP. Continuous MAP and HR data acquisition and analysis were done by using data acquisition software (LabChart, AD Instruments) with the Powerlab analog-to-digital convertor at a 1-kHz sampling rate. Basal measurements were obtained by taking the mean value over $30 \mathrm{~s}$ of baseline data immediately before nerve stimulation.

\section{Immunohistochemical Analysis for fDPN upon Repeated Electrical Stimulation}

At the end of the recording sessions, the animals were euthanized with an overdose of urethane. The fDPN tissue was collected, post-fixed, cryopreserved in $30 \%$ sucrose in $1 \times$ phosphatebuffered saline and cryosectioned at 20- $\mu \mathrm{m}$ sections for immunohistochemical analysis. Contralateral fDPN tissue was collected and used as a control (that is, no electrical stimulation). Potential stimulation-related acute axonal damage was evaluated by triple labeling immunofluorescence studies by using antibodies against the axonal marker $\beta$-III tubulin (1:500, mouse IgG; Sigma), myelin (P0) and activated macrophages (ED-1). The tissue sections were blocked with 5\% normal goat serum incubated overnight with the primary antibodies and reacted with $\mathrm{Cy} 2$ donkey anti-rabbit (1:250) and Cy3 donkey anti-mouse (1:500); Cy5 donkey anti-goat-stained (1:400) (Jackson labs) tissue sections were imaged by using an inverted Nikon Ti Eclipse confocal microscope with a $20 \times$ objective.

\section{Statistical Analyses}

MAP and HR from all the trials among different animals were expressed as mean \pm standard error of the mean. The data were analyzed by parametric twotailed Student $t$ test followed by MannWhitney post hoc evaluation using the Prism 4 software (GraphPad Software). Values with $p \leq 0.05$ were considered to be statistically significant.

\section{RESULTS}

\section{Cardiovascular Depressor Response by DPN Stimulation}

The baseline MAP and HR in normotensive WKY and hypertensive SHR rats was of $128.83 \pm 41$ and $149.75 \pm 35 \mathrm{mmHg}$ and $400.2 \pm 38$ and $350 \pm 83 \mathrm{bpm}$, respectively. Low-frequency hook-electrode stimulation of the DPN $(0.1 \mathrm{~mA}, 10$ pulses, 1-2 Hz) elicited a depressor response in both WKY $(-4.36 \mathrm{mmHg})$ and SHR $(-11.28 \mathrm{mmHg})$. The MAP reduction in these respective animals was more dramatic when $0.3 \mathrm{~mA}(-21.35$ and $-27.83 \mathrm{mmHg})$ and $0.5 \mathrm{~mA}(-39.64$ and $-33.52 \mathrm{mmHg}$ ) were used. Figure 2A shows the HR (top) and MAP (bottom) in an SHR rat in response to increasing 


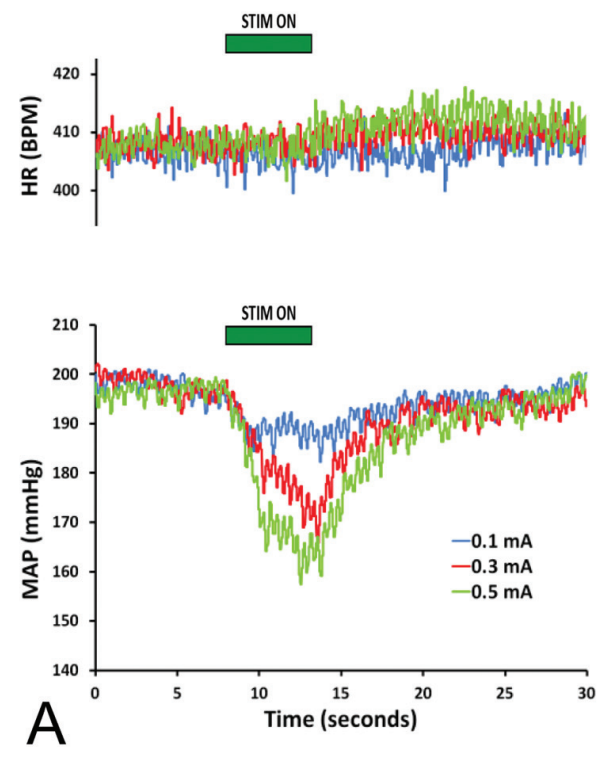

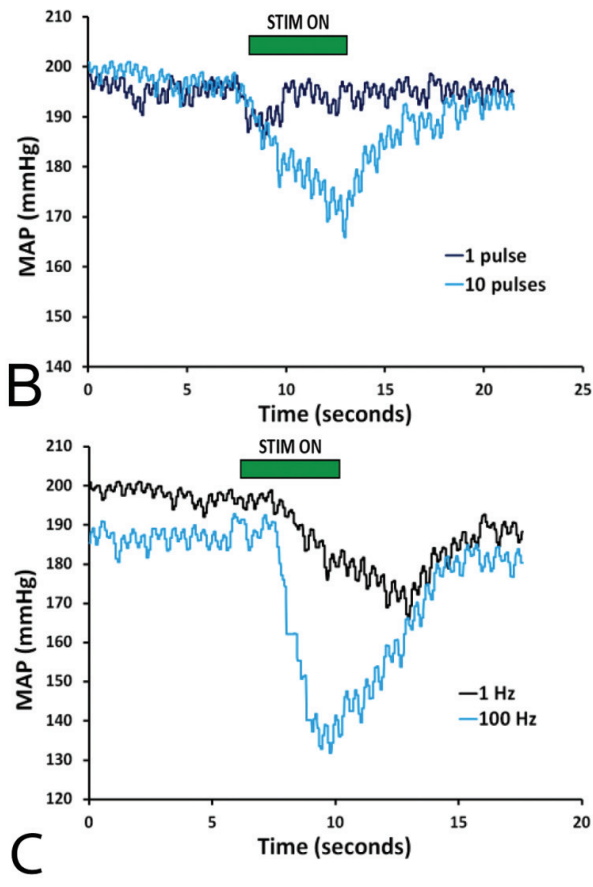

Figure 2. MAP depressor responses induced by DPN stimulation. Optimal stimulation parameters were evaluated in WKY and SHR animals. Representative MAP depressor responses after hook electrical stimulation (STIM) of the DPN nerve of an SHR animal.

(A) There was no effect on HR (top) and robust decrease in the MAP (bottom) in response to gradual increment in stimulus intensity under three different parameters. (B) MAP also decreases proportional to the number of stimulation pulses at $0.3 \mathrm{~mA}$ and $1-\mathrm{Hz}$ stimulation. (C) MAP depression in response to increased stimulation frequency from 1 to $100 \mathrm{~Hz}$ at $0.3 \mathrm{~mA}, 10$ pulses.

currents. While the higher stimulus intensity gave larger depressor responses, some muscle contraction was also noted at 0.3- and 0.5-mA intensities. We then used $0.5 \mathrm{~mA}$ at $1-2-\mathrm{Hz}$ stimulation parameters to evaluate the effect of pulse number and determined that increasing them from 1 to 10 potentiates the depressor response from -7.05 to -8.98 in WKY and from -33.52 to $-39.64 \mathrm{mmHg}$ in SHR (Figure 2B). Finally, we evaluated the effect of stimulation frequency using 10 pulses, at 0.3-mA current intensity. The comparison of 1-100 Hz showed an increment in the depressor response from -33.64 to $-40.45 \mathrm{mmHg}$ in WKY and -33.52 to $-50.84 \mathrm{mmHg}$ in SHR rats (Figure $2 \mathrm{C}$ ), respectively. These data showed that $0.1 \mathrm{~mA}$ in intensity, 10 pulses and $1-2-\mathrm{Hz}$ stimulation frequency are optimal to be used in fascicular fDPN stimulation using the $\mu \mathrm{CEA}$, since it achieves maximal depressor responses with minimal muscle activation.

\section{Significant Depressor Response by $\mu$ CEA Stimulation of the fDPN without Bradycardia}

When we tested short stimulation (that is, $5 \mathrm{~s}$ ) of the fDPN using the $\mu$ CEA electrode $(0.1 \mathrm{~mA}, 10$ pulses and $1 \mathrm{~Hz})$, the MAP did not change in normotensive rats (118.12 \pm 44.8 mmHg; $\mathrm{n}=6$; Figure 3A). In sharp contrast, SHR showed a maximum reduction in MAP to $126.2 \pm 33.3 \mathrm{mmHg}$ $(\mathrm{n}=6$; Figure 3B). This is a significant $23.6 \mathrm{mmHg}$ drop or a $15.8 \%$ reduction ( $p \leq 0.03$; Figure 3C). Figure 4 shows the individual response and variability for both HR and MAP responses in WKY and SHR animals (six animals per group) induced by electrical stimulation at fDPN. Baseline HR was similar between control and experimental animals with

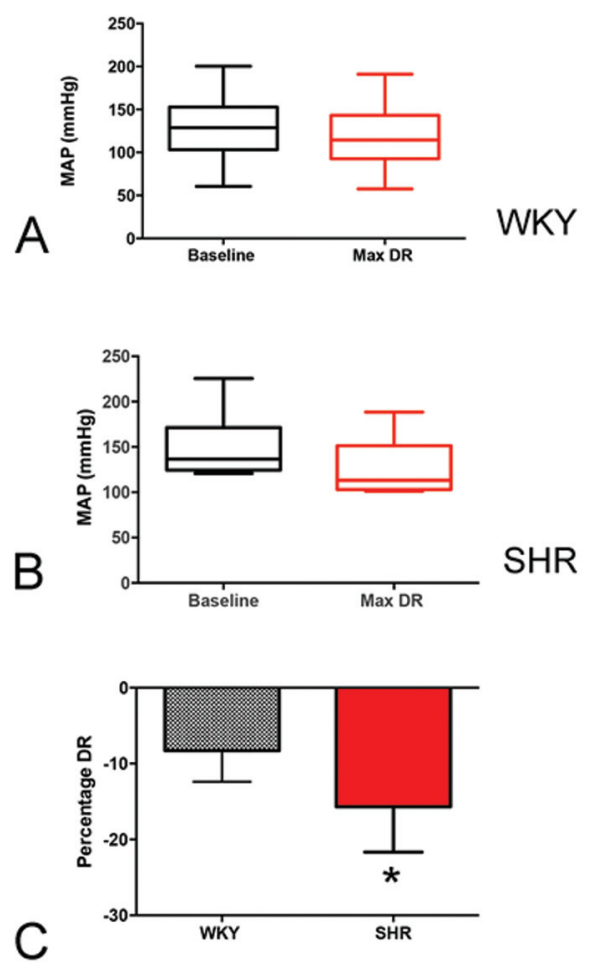

Figure 3. Mean MAP depressor responses after brief fDPN stimulation. A 5-s electrical stimulation of the fDPN did not significantly affect the average MAP in normotensive WKY animals (A), but induced a significant $(p<0.03)$ depressor response in SHR hypertensive animals of $\sim 10 \mathrm{mmHg}(B, C)$. The graphs show the mean and standard deviation from six animals in each group.

values of $400 \pm 37$ and $401 \pm 27$ beats per minute (bpm) in WKY and SHR, respectively (Figures 4A, C). Only one animal of the normotensive group showed a reduction in MAP in response to fascicular stimulation (Figure 4B). Conversely, all animals in the SHR group showed a depressor response elicited by stimulation of the fDPN fascicle. After the fDPN stimulation, the SHR returned to hypertensive levels in 5-15 s after stimulation (Figure 4D).

\section{Prolonged Depressor Response by Extended $\mu$ CEA Stimulation of the fDPN}

A separate cohort of animals $(n=3$, SHR) was used to evaluate the possibil- 

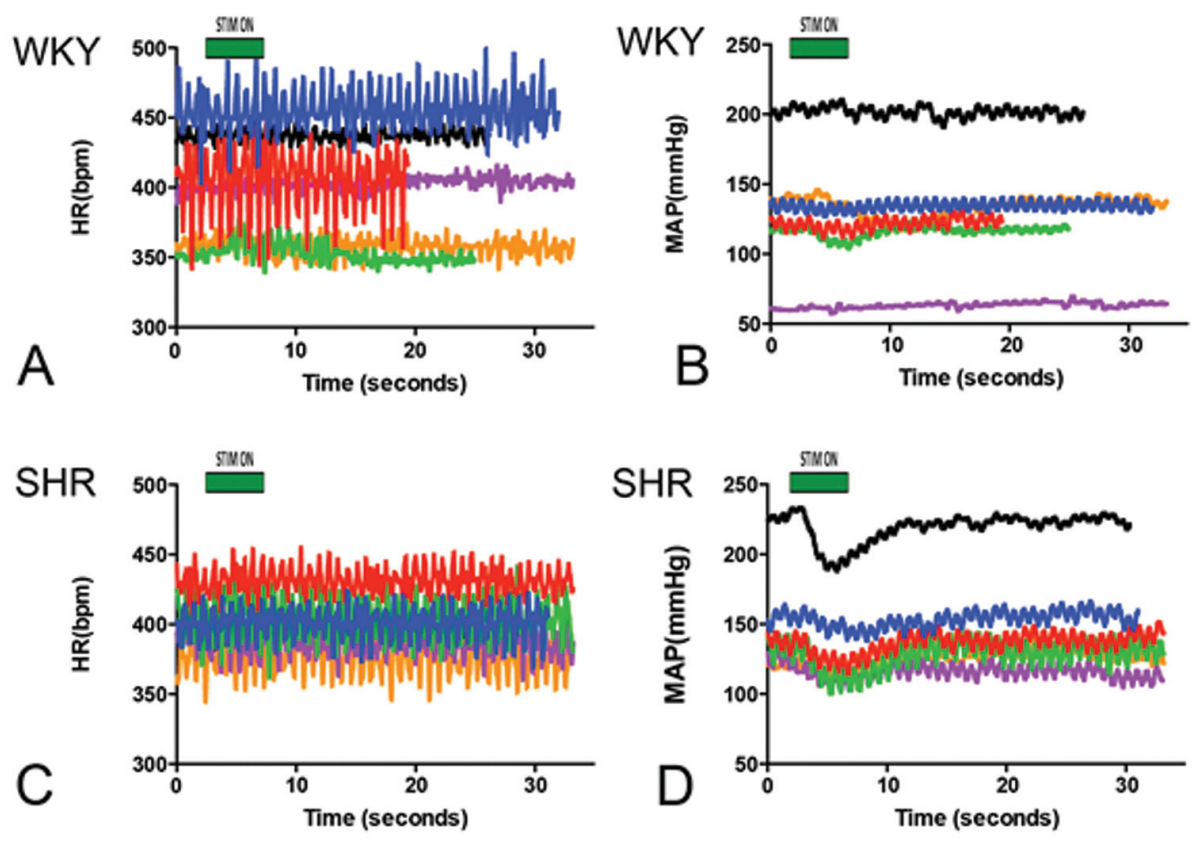

Figure 4. Individual MAP depressor responses in both WKY and SHR rats induced by short fDPN $\mu$ CEA electrical stimulation. HR and MAP recordings were elicited by 10 pulses (overlaying green trace; $0.1 \mathrm{~mA}, 1 \mathrm{~Hz}$ ) of electrical stimulation on the fDPN in six animals per group. No effect was observed on HR in either type of animal (A and C). The depressor response was observed in one WKY rat (green) (B) and in all SHR animals (D). This response returned to baseline values $15 \mathrm{~s}$ after stimulation.

ity of extending the depressor response by prolonging the stimulation from a few seconds to 5 min under the same current intensity and frequency. We observed an immediate reduction in MAP during the stimulation (Figure 5A), which persisted for up to $4 \mathrm{~h}$ in anesthetized animals (Figures 5B, C). Quantification of the MAP showed a reduction from baseline values $(181 \pm 6.5 \mathrm{mmHg})$ to $136 \pm 16$ and $141 \pm 8 \mathrm{mmHg}$ at 1 and $2 \mathrm{~h}$ after stimulation, respectively (Figure 6). Such sustained reduction in MAP represents a 24.86 to $17.12 \%$ decrease in blood pressure over a 2 -h period after the initial 5 -min stimulation. The data indicate a prolonged depressor response evoked by a short stimulation of the fDPN.

\section{Repeated Electrical Stimulation Does Not Induce Axonal Damage}

No obvious morphological nerve damage and no overt signs of trauma or edema/inflammation were observed during or after fascicular stimulation.
Qualitative immunohistochemical evaluation of the fDPN tissue after 5-min electrical stimulation showed normal histology. The NF-200-labeled axons were normal in morphology and label intensity (Figure 7A). No obvious damage to the myelin sheet was observed (Figure $7 \mathrm{~B})$, and no detection of activated macrophages (ED-1-positive cells) was noted (Figure 7C), suggesting that repetitive acute stimulation via $\mu \mathrm{CEA}$ did not cause overt damage to this fDPN tissue.

\section{DISCUSSION}

Refractory HTN is characterized by blood pressure that remains above $140 / 90 \mathrm{mmHg}$, despite treatments with at least two antihypertensive drugs. Among the surgical therapeutic strategies proposed are renal sympathetic denervation (18), carotid sinus baroreceptor stimulation $(19,20)$ and deep brain stimulation (21). All have shown some promise, but require complicated surgical interventions. Stimulation of autonomic nerves such as the vagal nerve or the carotid sinus nerve is particularly effective. However, these nerves are heterogeneous in afferent/efferent composition, and their stimulation often results in unwanted side effects such as bradycardia and bradypnea $(9,22)$.

In 1982, Yao et al. reported that electrical stimulation of somatic nerves might explain and reproduce the depressor effects of acupuncture in SHR rats. Indeed, acupuncture-like stimulation of the sciatic nerve in SHR animals was reported to induce an initial increase in HR and MAP during constant stimulation at $0.1 \mathrm{~mA}$, $3 \mathrm{~Hz}$ for $30 \mathrm{~min}$ and a subsequent decrease in MAP from 160 to $135 \mathrm{mmHg}$, which lasted several hours after stimulation (23). However, this stimulation paradigm also elicited leg twitches, and more specific stimulation strategies were needed. The ST36 acupoint receives sensory innervation from saphenous, superficial peroneal, and lateral sural cutaneous nerves and motor innervation from the deep peroneal and anterior tibialis nerves (24). Stimulation of the DPN for 8 wks has been shown to reduce MAP by $10-18 \mathrm{mmHg}$ without affecting HR (14). In humans, the peroneal nerve has approximately 25-38 fascicles (25) and whether stimulation of a single fascicle can significantly reduce MAP has not been evaluated. Here, we demonstrate that stimulating a single fascicle of the DPN (that is, fDPN) can be used to induce a depressor response in hypertensive SHR without significantly affecting HR. We showed that low-frequency stimulation $(1 \mathrm{~Hz})$ of 10 pulses at $0.1 \mathrm{~mA}$ induces an immediate MAP drop in a similar range to that observed after sciatic nerve stimulation or ST36-37 electroacupuncture, despite only interfacing a relatively small axonal population. The duration of the depressor response was significantly prolonged by extending the stimulation paradigm for $5 \mathrm{~min}$. This brief stimulation of the fDPN was found to be effective in reducing the MAP in SRH animals for up to $4 \mathrm{~h}$.

The mechanism of action of the observed depressor response in this study 

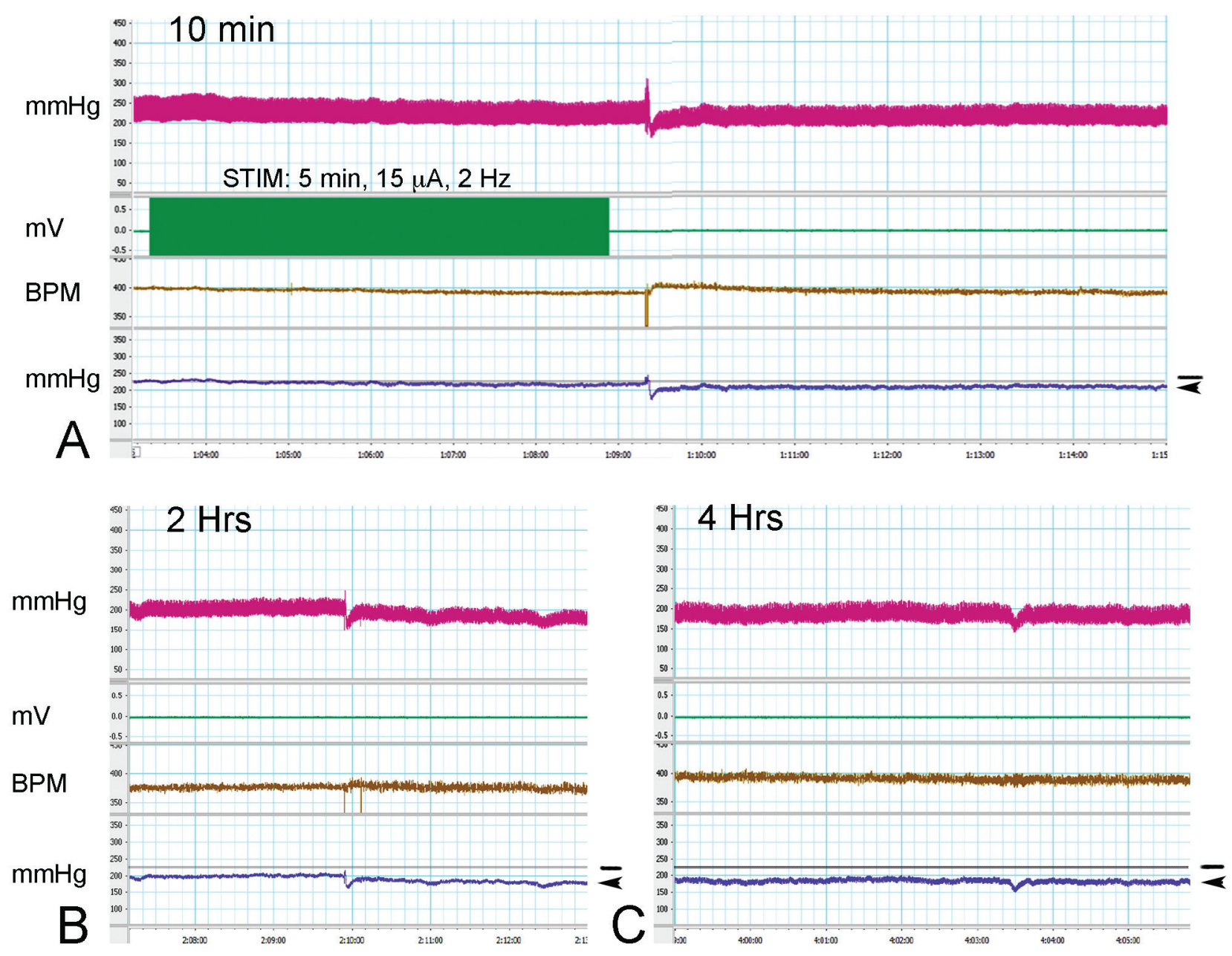

Figure 5. Prolonged MAP depressor response in SHR animals. (A) Representative traces of AP (arterial pressure, red), electrical stimulation (green), HR (brown) and MAP (blue) from a separate cohort of hypertensive rats that underwent 5 min of one-time fDPN stimulation, which produces a prolonged depressor response up to $4 \mathrm{~h}$. Arrowheads indicate the induced MAP levels compared with the baseline (black line) at $10 \mathrm{~min}(\mathrm{~A}), 2 \mathrm{~h}(\mathrm{~B})$ and $4 \mathrm{~h}(\mathrm{C})$ after stimulation (arrow heads). The MAP depressor response is evident shortly after stimulation and persists up to $4 \mathrm{~h}$.

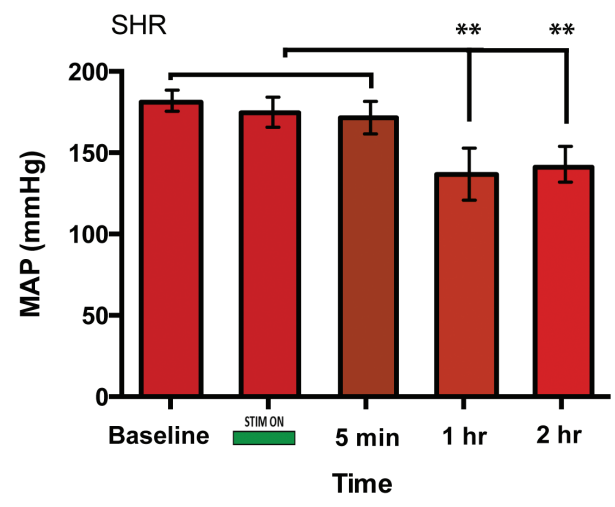

Figure 6. Maximum percentage in MAP depressor responses in SHR animals. Changes in MAP from SRH rats $(n=3)$, which was significantly reduced at both 1 and $2 \mathrm{~h}$ after the initial 5 min stimulation. ${ }^{* *} p<0.001$.

remains to be elucidated. However, the acupuncture stimulation of the ST36 acupoint has been shown to stimulate the DPN and to evoke neural afferent activity from the skin, muscles and joints. These axons terminate in the dorsal horn of the lumbar spinal cord, where they activate GABA-ergic interneurons, which in turn mediate synaptic inhibition of second-order neurons projecting to the brain stem periaqueductal gray, hypothalamic nuclei and rostral ventrolateral medulla (26) regions. Thus, a complex heterosynaptic pathway is likely involved in eliciting the hypotensive effect 

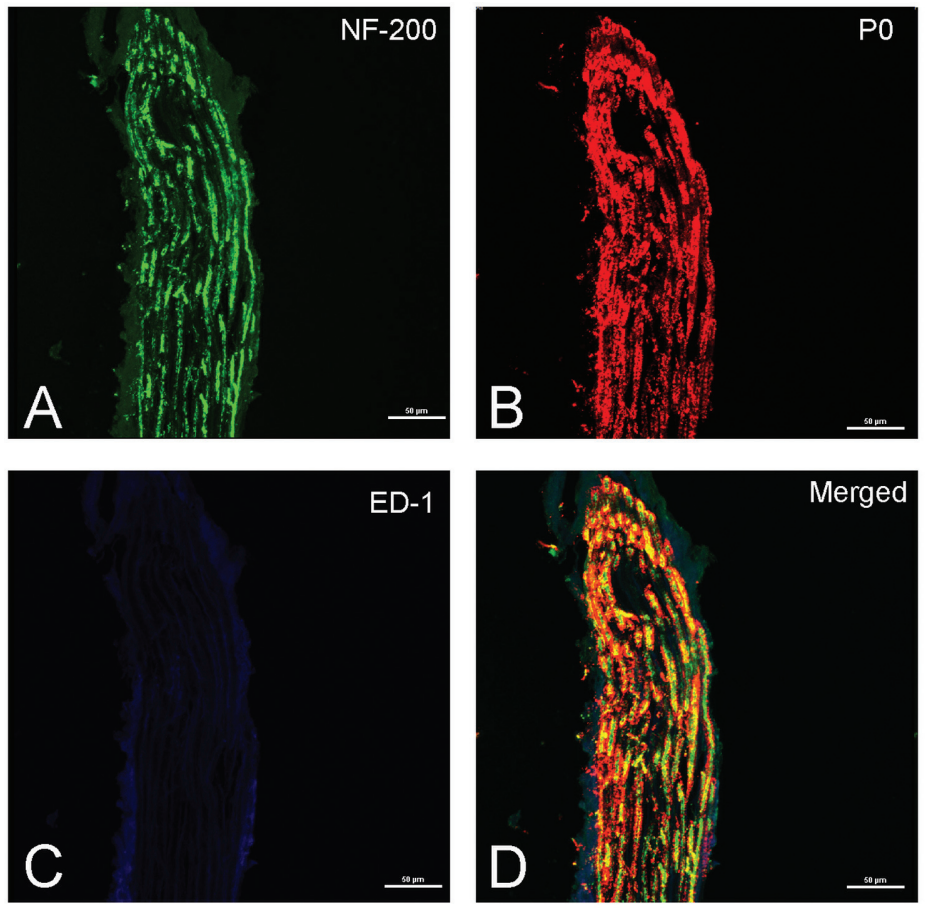

Figure 7. Normal axonal histology in fDPN tissue after 5 min of $\mu$ CEA electrical stimulation. (A) Representative peroneal nerve fascicle from the animals that were subjected to 5-min stimulation and 4-h monitoring were labeled with the axonal (NF-200, green) myelination (PO, red) and inflammation (ED-1, blue) antibodies. Normal histology was noted, and no overt nerve damage or inflammation was observed. Scale bar $=50 \mu \mathrm{m}$.

induced by stimulation of this nerve. This result has been confirmed by recent studies that have demonstrated that damage to the DPN abolishes the depressor response (27). Others have reported that DPN stimulation at $12 \mathrm{~Hz}$ and $0.15-0.3 \mathrm{~mA}$ excites only $\mathrm{A} \alpha, \mathrm{A} \beta$ and $\mathrm{A} \delta$ fibers, whereas $0.5-0.8 \mathrm{~mA}$ evokes activity in C-fibers (28). Thus, it is likely that the effect that others and we have observed relates to the autonomic regulation of vasodilation in the hind limb.

One of the limitations of the present study is that all experiments were done in anesthetized animals, and thus further studies are warranted to determine whether the fDPN stimulation can be achieved in conscious animals. Such experiments will require not only the use of microelectrode arrays such as the $\mu \mathrm{CEA}$, but also the development of fully implantable systems. We are currently working on the development of wireless stimulating microelectrodes, which can be used in the future to evaluate the effect of fDPN stimulation in fully awake animals.

\section{CONCLUSION}

In summary, this study provides evidence supporting the use of $\mu \mathrm{CEA}$ interfacing of small somatic nerve fascicles associated with cardiovascular relevant acupoints to induce significant reductions in MAP. The findings open the possibility of clinically relevant neuromodulation of small fascicles, which can be used as a strategy to achieve selective R-HTN therapy with minimal side effects.

This study was supported by the Texas Medical Research Collaborative Fund (TxMRC; MI Romero-Ortega) and the University of Texas at Arlington Research Institute (UTARI) at UT Arlington.

\section{ACKNOWLEDGMENTS}

\section{DISCLOSURE}

The authors declare that they have no competing interests as defined by Bioelectronic Medicine, or other interests that might be perceived to influence the results and discussion reported in this paper.

\section{REFERENCES}

1. Badheka AO, et al. (2015) Trends of hospitalizations in the United States from 2000 to 2012 of patients $>60$ years with aortic valve disease. $A m$. J. Card. 116:132-41.

2. James PA, et al. (2014) Evidence-based guideline for the management of high blood pressure in adults: report from the panel members appointed to the Eighth Joint National Committee (JNC 8). JAMA. 311:507-20.

3. Malagutti N. (2014) Particle filter-based robust adaptive control for closed-loop administration of sodium nitroprusside. J. Comput. Surg. 1:8

4. Volpe M, de la Sierra A, Kreutz R, Laurent S, Manolis AJ. (2014) ARB-based single-pill platform to guide a practical therapeutic approach to hypertensive patients. High Blood Press. Cardiovasc. Prev. 21:137-47.

5. Calhoun DA, et al. (2008) Resistant hypertension: diagnosis, evaluation, and treatment: a scientific statement from the American Heart Association Professional Education Committee of the Council for High Blood Pressure Research. Hypertension. 51:1403-19.

6. Ott C, Schmieder RE. (2014) Invasive treatment of resistant hypertension: present and future. Curr. Hypertens. Rep. 16:488.

7. Bhatt DL, et al. (2014) A controlled trial of renal denervation for resistant hypertension. N. Engl. J. Med. 370:1393-401.

8. Fahy BG. (2010) Intraoperative and perioperative complications with a vagus nerve stimulation device. J. Clin. Anesth. 22:213-22.

9. Plachta DT, et al. (2014) Blood pressure control with selective vagal nerve stimulation and minimal side effects. J. Neural. Eng. 11:036011.

10. Ben-Menachem E. (2001) Vagus nerve stimulation, side effects, and long-term safety. J. Clin. Neurophysiol. 18:415-8.

11. Flachskampf FA, et al. (2007) Randomized trial of acupuncture to lower blood pressure. Circulation. 115:3121-9.

12. Li DZ, et al. (2014) Acupuncture for essential hypertension: a meta-analysis of randomized shamcontrolled clinical trials. Evid. Based Complement. Alternat. Med. 2014:279478.

13. Hoffmann P, Thoren P. (1986) Long-lasting cardiovascular depression induced by acupuncturelike stimulation of the sciatic nerve in unanaesthetized rats: effects of arousal and type of hypertension. Acta Physiol. Scand. 127:119-26.

14. Li P, Longhurst JC. (2010) Neural mechanism of electroacupuncture's hypotensive effects. Auton. Neurosci. 157:24-30. 
15. Kim Y, et al. (c2014) A novel microchannel electrode array: towards bioelectronic medical interfacing of small peripheral nerves. In: Engineering in Medicine and Biology Society (EMBC), 2014 36th Annual International Conference of the IEEE; 2014 Aug 24-30; Chicago (IL). p. 1981-4. Available from: http: / /ieeexplore.ieee.org/xpl/freeabs_all.jsp? arnumber $=6944002 \& \operatorname{tag}=1 \&$ abstractAccess $=$ no \&userType=inst

16. Okamoto K, Aoki K. (1963) Development of a strain of spontaneously hypertensive rats. Jpn. Circ. J. 27:282-93.

17. Mizuno M, Murphy MN, Mitchell JH, Smith SA. (2011) Skeletal muscle reflex-mediated changes in sympathetic nerve activity are abnormal in spontaneously hypertensive rats. Am. J. Physiol. 300:H968-77.

18. Krum H, et al. (2009) Catheter-based renal sympathetic denervation for resistant hypertension: a multicentre safety and proof-of-principle cohort study. Lancet. 373:1275-81.

19. Wustmann K, et al. (2009) Effects of chronic baroreceptor stimulation on the autonomic cardiovascular regulation in patients with drug-resistant arterial hypertension. Hypertension. 54:530-6.

20. Illig KA, et al. (2006) An implantable carotid sinus stimulator for drug-resistant hypertension: surgical technique and short-term outcome from the multicenter phase II Rheos feasibility trial. J. Vasc. Surg. 44:1213-8.

21. Pereira EAC, et al. (2010) Sustained reduction of hypertension by deep brain stimulation. J. Clin. Neurosci. 17:124-7.

22. Sun X, Lan Q-q, Cai Y, Yu Y-q. (2012) Electrical stimulation of deep peroneal nerve mimicking acupuncture inhibits the pressor response via capsaicin-insensitive afferents in anesthetized rats. Chin. J. Integr. Med. 18:130-6.

23. Yao T, Andersson S, Thoren P. (1982) Longlasting cardiovascular depression induced by acupuncture-like stimulation of the sciatic nerve in unanaesthetized spontaneously hypertensive rats. Brain Res. 240:77-85.

24. Xia Y. (2010) Acupuncture therapy for neurological diseases: a neurobiological view. Beijing, China: Tsinghua University Press, Springer.

25. Tompkins RP, Melling CW, Wilson TD, Bates BD, Shoemaker JK. (2013) Arrangement of sympathetic fibers within the human common peroneal nerve: implications for microneurography. J. Appl. Physiol. 115:1553-61.

26. Zhou SY, Zhu YP, Gao F. (2003) The regulation of deep peroneal nerve inputs on cardiovascular activity caused by area postrema excitation [in Chinese]. Zhongguo Ying Yong Sheng Li Xue Za Zhi. 19:7, 29.

27. Quiroz-Gonzalez S, Segura-Alegria B, JimenezEstrada I. (2014) Depressing effect of electroacupuncture on the spinal non-painful sensory input of the rat. Exp. Brain Res. 232:2721-9.

28. Ohsawa H, Okada K, Nishijo K, Sato Y. (1995) Neural mechanism of depressor responses of arterial pressure elicited by acupuncture-like stimulation to a hindlimb in anesthetized rats. Journal of the Autonomic Nervous System. 51:27-35.
Cite this article as: Kim Y, et al. (2015) Microchannel electrode stimulation of deep peroneal nerve fascicles induced mean arterial depressor response in hypertensive rats. Bioelectron. Med. 2:55-62. 